\title{
Spin-liquid versus spiral-order phases in the anisotropic triangular lattice
}

\author{
Luca F. Tocchio, ${ }^{1}$ Hélène Feldner, ${ }^{1}$ Federico Becca, ${ }^{2}$ Roser Valentí, ${ }^{1}$ and Claudius Gros ${ }^{1}$ \\ ${ }^{1}$ Institute for Theoretical Physics, University of Frankfurt, \\ Max-von-Laue-Straße 1, D-60438 Frankfurt a.M., Germany \\ ${ }^{2}$ CNR-IOM-Democritos National Simulation Centre and International School \\ for Advanced Studies (SISSA), Via Bonomea 265, I-34136, Trieste, Italy
}

(Dated: July 13, 2018)

\begin{abstract}
We study the competition between magnetic and spin-liquid phases in the Hubbard model on the anisotropic triangular lattice, which is described by two hopping parameters $t$ and $t^{\prime}$ in different spatial directions and is relevant for layered organic charge-transfer salts. By using a variational approach that includes spiral magnetic order, we provide solid evidence that a spin-liquid phase is stabilized in the strongly-correlated regime and close to the isotropic limit $t^{\prime} / t=1$. Otherwise, a magnetically ordered spiral state is found, connecting the (collinear) Néel and the (coplanar) $120^{\circ}$ phases. The pitch vector of the spiral phase obtained from the unrestricted Hartree-Fock approximation is substantially renormalized in presence of electronic correlations, and the Néel phase is stabilized in a wide regime of the phase diagram, i.e., for $t^{\prime} / t<0.75$. We discuss these results in the context of organic charge-transfer salts.
\end{abstract}

PACS numbers: 71.10.Fd, 71.27.+a, 75.10.-b

Introduction- The combined presence of strong electron interaction and geometrical frustration leads to a plethora of interesting phenomena, like superconductivity, metal-insulator (Mott) transition, or purely quantum paramagnets, the so-called spin liquids. In this context, the organic charge-transfer salts $\kappa$-(ET) $)_{2} \mathrm{X}$ [1] play an important role. 2 24] A large variety of phases have been found when changing temperature, pressure or the nature of the anion X, ranging from correlated metals with superconductivity at low temperatures, to insulators with magnetic order. [5 8] Even more interestingly, a metalinsulator transition to a pure non-magnetic Mott insulating state has been detected for the compound with $\mathrm{X}=\mathrm{Cu}_{2}(\mathrm{CN})_{3}$. 9, 10] Recently, another family of organic materials, denoted by $\mathrm{Et}_{n} \mathrm{Me}_{4-n} P n\left[\mathrm{Pd}(\mathrm{dmit})_{2}\right]_{2}$, [11] has been shown to display different quantum phases, including valence-bond solid and spin-liquid states. 12, 13. From a quantum chemical perspective, the simplest possible effective Hamiltonian for the organic charge-transfer salts is the Hubbard model (after an appropriate particlehole transformation) on the anisotropic triangular lattice at half filling. Indeed, in these materials, strongly dimerized organic molecules are arranged in stacked twodimensional triangular lattices; each dimer has (on the average) a charge state with one hole, implying a halffilled conducting band. In addition, a sizable effective Coulomb repulsion is felt by two holes on the same dimer, while longer range correlations are much smaller. [14, 15]

The Hubbard model is defined by:

$$
\mathcal{H}=-\sum_{i, j, \sigma} t_{i j} c_{i, \sigma}^{\dagger} c_{j, \sigma}+\text { h.c. }+U \sum_{i} n_{i, \uparrow} n_{i, \downarrow}
$$

where $c_{i, \sigma}^{\dagger}\left(c_{i, \sigma}\right)$ creates (destroys) an electron with spin $\sigma$ on site $i, n_{i, \sigma}=c_{i, \sigma}^{\dagger} c_{i, \sigma}$ is the electronic density, $t_{i j}$ is the hopping amplitude and $U$ is the on-site Coulomb
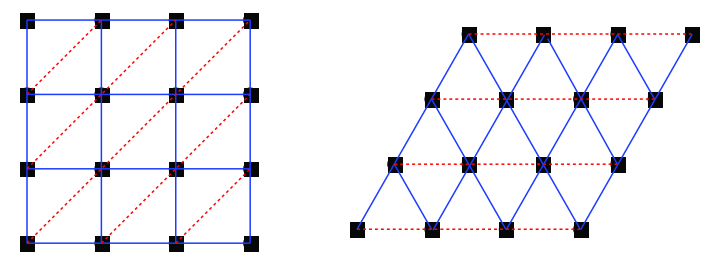

FIG. 1: Illustration of the anisotropic triangular lattice in the square topology (left), used in this work, and in the equivalent triangular topology (right). Solid and dashed lines denote hopping amplitudes $t$ and $t^{\prime}$, respectively.

repulsion. In this work, we focus our attention on the half-filled case, where the number of electrons $N_{e}$ equals the number of sites $L$, and consider a square lattice with a nearest-neighbor hopping $t$, along $(1,0)$ and $(0,1)$ directions, and a further next-nearest-neighbor hopping $t^{\prime}$ along $(1,1)$; this choice of the hopping amplitudes is topologically equivalent to the anisotropic triangular lattice, see Fig. 1. According to recent density functional theory calculations, [14 17] the ratio $t^{\prime} / t$ appropriate for organic salts lies in the range $[0.3,1.3]$, with the spin-liquid compound $\kappa-(\mathrm{ET})_{2} \mathrm{Cu}_{2}(\mathrm{CN})_{3}$ located at $t^{\prime} / t \simeq 0.83$.

A major issue in the Hubbard model on the anisotropic triangular lattice is the possibility of stabilizing a spinliquid phase, compatible with the experimental data. From one side, several approaches have proposed the existence of a spin liquid-region for $t^{\prime} / t<1$, based on path-integral renormalization group (PIRG), [18] dynamical mean-field theory (DMFT), [19] exact diagonalization, 20] and variational Monte Carlo (VMC). 21] In addition, several studies suggested a possible spin-liquid phase even for the isotropic case $t^{\prime} / t=1$, close to the metal-insulator transition. 22 25] From the other side, for generic values of the ratio $t^{\prime} / t$, magnetic states with incommensurate order may be expected and indeed have 
been proposed by different mean-field approaches, like for instance within the Hartree-Fock (HF) approximation 26 28] or the renormalized mean-field method. 29] However, due to the difficulty of constructing correlated magnetic states with generic ordering vectors, none of the previous studies was able to perform a fair comparison between spin-liquid and spiral states. In this respect, some progress to deal with incommensurate magnetism in the Heisenberg model has been done by means of analytic approximations, 30 32 or density-matrix renormalization group (DMRG) calculations, 33] even if here the long-range nature of the magnetic correlations is not addressed. Instead, incommensurate correlations in the Hubbard model have been only marginally addressed by Cluster-DMFT. 34]

In order to go beyond the previous studies, we approach this problem by implementing correlated variational wave functions which describe magnetic states with generic incommensurate order. This can be achieved by starting from the spiral states obtained at the HF level and including, in a second step, many body correlations. In this way, we are able to treat incommensurate spiral order and non-magnetic states on the same level and determine which state is stabilized for a given value of frustration $t^{\prime} / t$ and Coulomb repulsion $U$. Variational approaches may contain, as a matter of principle, a bias towards ordered states. However, we showed previously 21, 35] that very accurate results are obtained in a wide regime of the parameters when using, as in the present work, generalized Gutzwiller wave functions with long-range Jastrow correlations and backflow corrections.

Here, we confirm that a spin-liquid phase is favored for $t^{\prime} / t \simeq 0.85$, while magnetic spiral order becomes competitive close to the isotropic point, i.e., for $t^{\prime} / t \simeq 0.95$, and for $t^{\prime} / t \simeq 0.75-0.8$. In addition, we explicitly study the effect of correlations on a mean-field state showing how the energy and the pitch vector of the spiral state are modified when electronic correlations are taken into account by the VMC method.

Unrestricted Hartree Fock- The unrestricted HF state is obtained by performing a mean-field decoupling of Eq. (1):

$$
\begin{aligned}
H_{\mathrm{HF}}= & -\sum_{i, j, \sigma} t_{i j} c_{i, \sigma}^{\dagger} c_{j, \sigma}+\text { h.c. } \\
& +U \sum_{i}\left[\left\langle n_{i, \downarrow}\right\rangle n_{i, \uparrow}+\left\langle n_{i, \uparrow}\right\rangle n_{i, \downarrow}\right] \\
& -U \sum_{i}\left[\left\langle c_{i, \uparrow}^{\dagger} c_{i, \downarrow}\right\rangle c_{i, \downarrow}^{\dagger} c_{i, \uparrow}+\left\langle c_{i, \downarrow}^{\dagger} c_{i, \uparrow}\right\rangle c_{i, \uparrow}^{\dagger} c_{i, \downarrow}\right] \\
& -U \sum_{i}\left[\left\langle n_{i, \uparrow}\right\rangle\left\langle n_{i, \downarrow}\right\rangle-\left\langle c_{i, \uparrow}^{\dagger} c_{i, \downarrow}\right\rangle\left\langle c_{i, \downarrow}^{\dagger} c_{i, \uparrow}\right\rangle\right],
\end{aligned}
$$

which contains $4 L$ independent mean-field parameters to be computed self-consistently: $\left\langle n_{i, \uparrow}\right\rangle,\left\langle n_{i, \downarrow}\right\rangle,\left\langle c_{i, \uparrow}^{\dagger} c_{i, \downarrow}\right\rangle$, and $\left\langle c_{i, \downarrow}^{\dagger} c_{i, \uparrow}\right\rangle$ for each site. Here, we slightly restrict
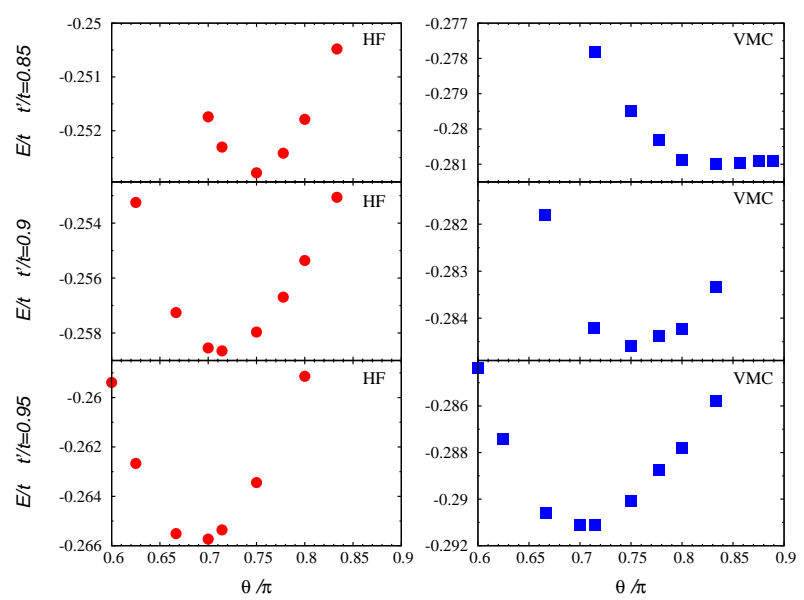

FIG. 2: (Color online) HF (left) and VMC (right) energies of the spiral state, for $U / t=16$, as a function of the pitch angle $\theta$ (in unit of $\pi$ ), for $t^{\prime} / t=0.85$ (top), 0.9 (middle) and 0.95 (bottom). Cluster of sizes $l \times l$ have been used, with $l=12,14,16,18,20$.

the variational freedom and impose the spin order to be coplanar in the $x-y$ plane, namely, we look for solutions with $\left\langle n_{i, \uparrow}\right\rangle=\left\langle n_{i, \downarrow}\right\rangle$, thus reducing the number of independent parameters to $3 L$. The mean-field Hamiltonian (2) can be diagonalized and the ground state $|\mathrm{SP}\rangle$ can be computed by filling the lowest-energy singleparticle orbitals.

Here, we are interested in describing the nature of the insulating state, which is stabilized for sufficiently large on-site interactions. In this regime, the optimal HF solutions display a spiral magnetic order, which, for $t^{\prime} / t \leq 1$, may be parametrized through a single pitch angle $\theta \in[2 \pi / 3, \pi]$. Indeed, nearest-neighbor spins, along $(1,0)$ and $(0,1)$ directions, form an angle $\theta$, while nextnearest-neighbor spins, along the $(1,1)$ direction, form an angle $2 \theta$; a pitch angle of $\theta=\pi$ corresponds to Néel order, suitable for $t^{\prime}=0$, and $\theta=2 \pi / 3$ to the $120^{\circ}$ order, suitable for $t^{\prime}=t$. On finite-size clusters with periodicboundary conditions, only the set of commensurate pitch angles is accessible; for $L=l \times l$, the allowed values are $\theta=2 \pi n / l$, with $n$ being an integer.

Variational Monte Carlo- Within the VMC approach, we construct magnetic states with spiral order by applying correlation terms on top of spiral states. Since the optimal pitch angle in the presence of electron correlations may differ from the one obtained at the $\mathrm{HF}$ level, several different values of $\theta$ are considered in the VMC calculations. We employ a spin-spin Jastrow factor to correctly describe fluctuations orthogonal to the plane where the magnetic order lies, i.e., $\mathcal{J}_{s}=\exp \left[1 / 2 \sum_{i, j} u_{i, j} S_{i}^{z} S_{j}^{z}\right]$. [36] A further densitydensity Jastrow factor $\mathcal{J}_{c}=\exp \left[1 / 2 \sum_{i, j} v_{i, j} n_{i} n_{j}\right]$ (that includes the on-site Gutzwiller term $v_{i, i}$ ) is considered to adjust electron correlations. All the $u_{i, j}$ 's and the $v_{i, j}$ 's 


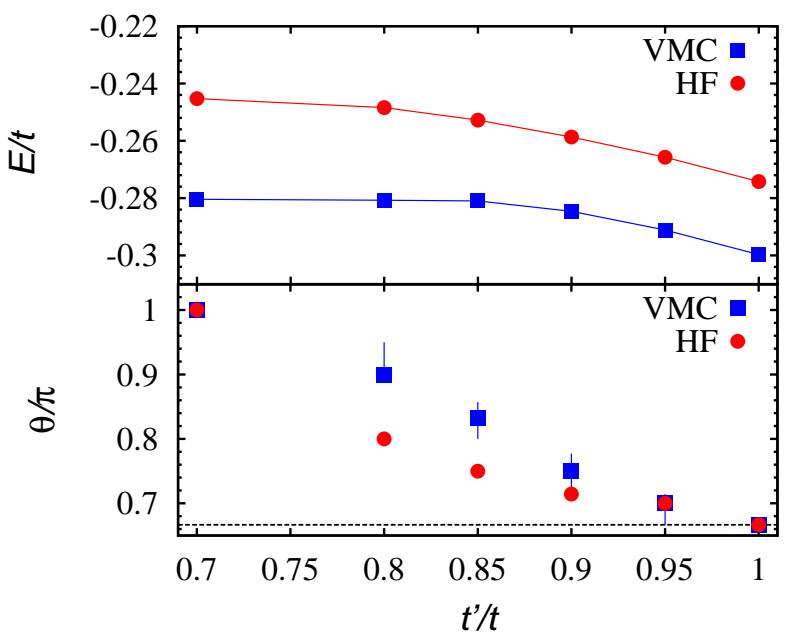

FIG. 3: (Color online) Upper panel: HF (red circles) and VMC (blue squares) energies of the optimal spiral state as a function of $t^{\prime} / t$ for $U / t=16$. Lower panel: the pitch angle $\theta$ (in unit of $\pi$ ) of the optimal spiral state as a function of $t^{\prime} / t$ for $U / t=16$. The dotted horizontal line corresponds to $\theta=2 \pi / 3$, suitable for the isotropic point $t^{\prime} / t=1$. The error bars in the VMC calculations are due to the finite-size limitations in the accessible pitch angles.

are optimized for every independent distance $|i-j|$. The correlated state is then given by $\left|\Psi_{\mathrm{SP}}\right\rangle=\mathcal{J}_{s} \mathcal{J}_{c}|\mathrm{SP}\rangle$.

In order to describe a non-magnetic insulator we construct, in a first step, an uncorrelated wave function given by the ground state $|\mathrm{BCS}\rangle$ of a superconducting BCS Hamiltonian: 37 40]

$$
\mathcal{H}_{\mathrm{BCS}}=\sum_{k, \sigma} \xi_{k} c_{k, \sigma}^{\dagger} c_{k, \sigma}+\sum_{k} \Delta_{k} c_{k, \uparrow}^{\dagger} c_{-k, \downarrow}^{\dagger}+\text { h.c. }
$$

where both the free-band dispersion $\xi_{k}$ and the pairing amplitudes $\Delta_{k}$ are variational functions. We use the parametrization

$$
\begin{aligned}
\xi_{k} & =-2 \tilde{t}\left(\cos k_{x}+\cos k_{y}\right)-2 \tilde{t}^{\prime} \cos \left(k_{x}+k_{y}\right)-\mu \\
\Delta_{k} & =2 \Delta_{\mathrm{BCS}}\left(\cos k_{x}-\cos k_{y}\right)
\end{aligned}
$$

where the effective hopping amplitude $\tilde{t}^{\prime}$, the effective chemical potential $\mu$, and the pairing field $\Delta_{\mathrm{BCS}}$ are variational parameters to be optimized. The $d$-wave symmetry of the pairing function introduced in Eq. (5) is found to be the best variational state in all the range $t^{\prime} / t \leq 1$. The correlated state $\left|\Psi_{\mathrm{BCS}}\right\rangle=\mathcal{J}_{c}|\mathrm{BCS}\rangle$ allows then to describe a non-magnetic Mott insulator for a sufficiently singular Jastrow factor $v_{q} \sim 1 / q^{2}\left(v_{q}\right.$ being the Fourier transform of $v_{i, j}$ ). [41]

A size-consistent and efficient way to further improve the correlated states $\left|\Psi_{\mathrm{BCS}}\right\rangle$ and $\left|\Psi_{\mathrm{SP}}\right\rangle$ is based on backflow correlations. In this approach, each orbital that defines the unprojected states $|\mathrm{BCS}\rangle$ and $|\mathrm{SP}\rangle$ is taken to depend upon the many-body configuration, in order

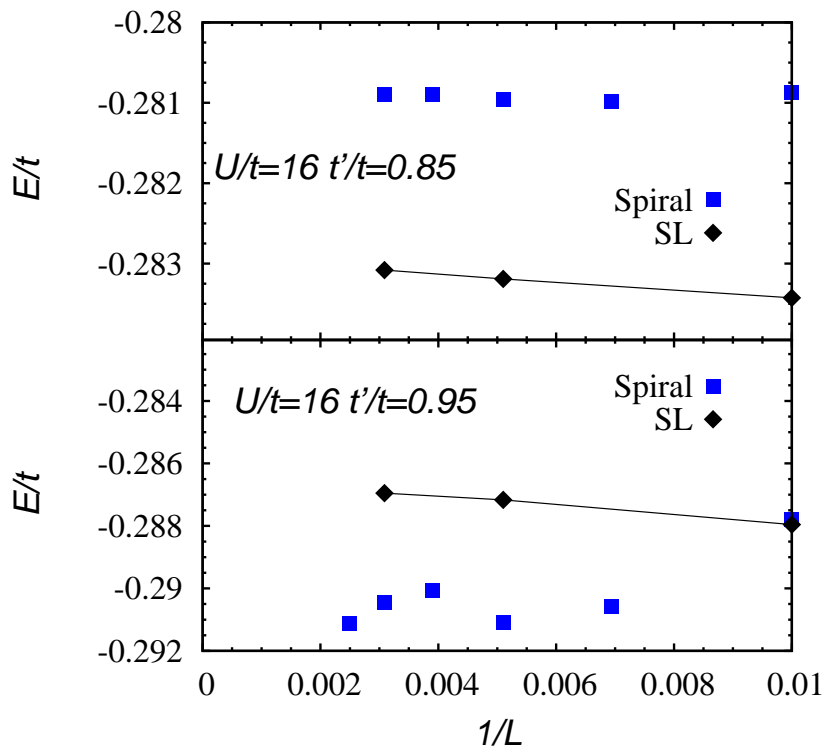

FIG. 4: (Color online) Upper panel: VMC energy of the optimal spiral (blue squares) and spin-liquid (black diamonds) states as a function of the inverse system size $1 / L$, with $L$ ranging from $10 \times 10$ to $20 \times 20$. Data refer to the case $t^{\prime} / t=0.85$ and $U / t=16$. Lower panel: the same as in the upper panel but for $t^{\prime} / t=0.95$.

to incorporate virtual hopping processes. 35] All results presented here are obtained by fully incorporating the backflow corrections and optimizing individually every variational parameter in $\xi_{k}$ and $\Delta_{k}$, in the Jastrow factors $\mathcal{J}_{c}$ and $\mathcal{J}_{s}$, as well as in the backflow corrections.

Finally, we want to mention that the $d$-wave symmetry of the pairing function, introduced in Eq. (5), is in agreement with previous VMC studies [42, 43] carried out using variational wave functions not containing longrange Jastrow and backflow correlations.

Evolution of the pitch angle- In Fig. 2] the energy per site of the spiral state is presented as a function of the pitch angle $\theta$, for different values of the frustrating hoppings $t^{\prime} / t$ and $U / t=16$, both for the HF and the VMC calculations. We mention that the pitch angle is only weakly dependent on $U / t$ in the insulating region. For finite lattices the set of allowed pitch angles is determined by commensurability, we therefore include in Fig. 2 results for several cluster sizes. The overall behavior of the energy per site versus $\theta$ is smooth, indicating that size effects are under control. In the region of $0.8 \lesssim t^{\prime} / t \lesssim 0.9$, a very shallow energy landscape is observed in VMC, while, for larger values of the frustrating hopping, the minimum is much more pronounced.

We find that Jastrow and backflow terms influence the periodicity of the spiral order and that the inclusion of the correlation factors induce a sizable gain in the energy per site, strongly improving the quality of the variational state. In Fig. 3, we present the evolution of the energy 


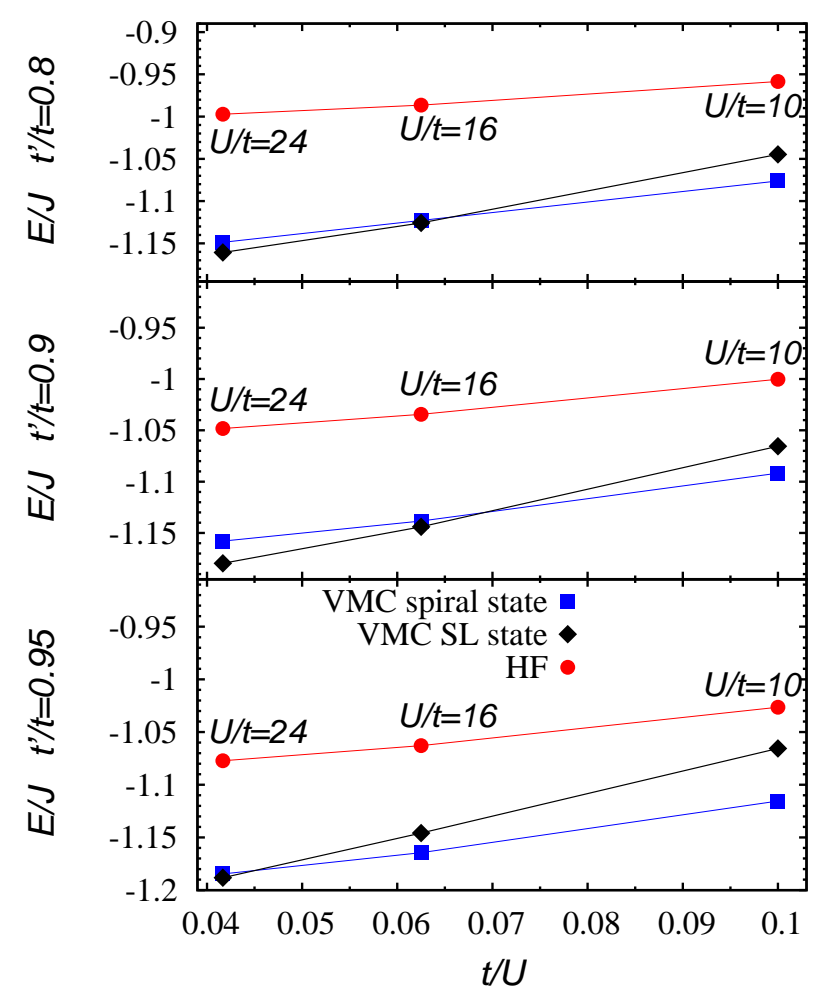

FIG. 5: (Color online) The energy (in unit of $J=4 t^{2} / U$ ) as a function of $t / U$ for the HF approximation (red circles), the optimal spiral state in VMC (blue squares), and the spinliquid state (black diamonds) for $t^{\prime} / t=0.8$ (top), 0.9 (middle) and 0.95 (bottom).

and of the pitch angle $\theta$ of the optimal spiral state as a function of $t^{\prime} / t$, for $U / t=16$. In the intermediate range of frustration $0.75 \lesssim t^{\prime} / t \lesssim 0.9$, the explicit treatment of electronic correlations (within the VMC level) renormalizes the angle of the HF optimal spiral state and values much closer to $\pi$ are stabilized. As a result, correlation effects stabilize the Néel phase (i.e., $\theta=\pi$ ) in a wider regime, e.g., for $t^{\prime} / t<0.75$. Close to the isotropic point, i.e., for $t^{\prime} / t \lesssim 1.0$, the optimal pitch angle shifts rapidly towards $2 \pi / 3$.

Spiral vs. spin-liquid state- Let us now move to the main result of the present work and compare the optimal spiral and spin-liquid states. We find that for $t^{\prime} / t=0.85$ and $U / t=16$, which are suitable parameters for $\kappa$ - $(\mathrm{ET})_{2} \mathrm{Cu}_{2}(\mathrm{CN})_{3}$, the lowest variational energy is achieved by a magnetically disordered state, see Fig. 4 . The results are only weakly dependent on the cluster size. On the other hand, for a larger value of the frustrating hopping, i.e., $t^{\prime} / t=0.95$, a spiral state with angle $\theta / \pi \simeq 0.7$ is favored over the spin liquid. In this case, although slightly larger size effects are present for the spiral state, the trend is clear.

In Fig. 5] we present the VMC energies (in unit of

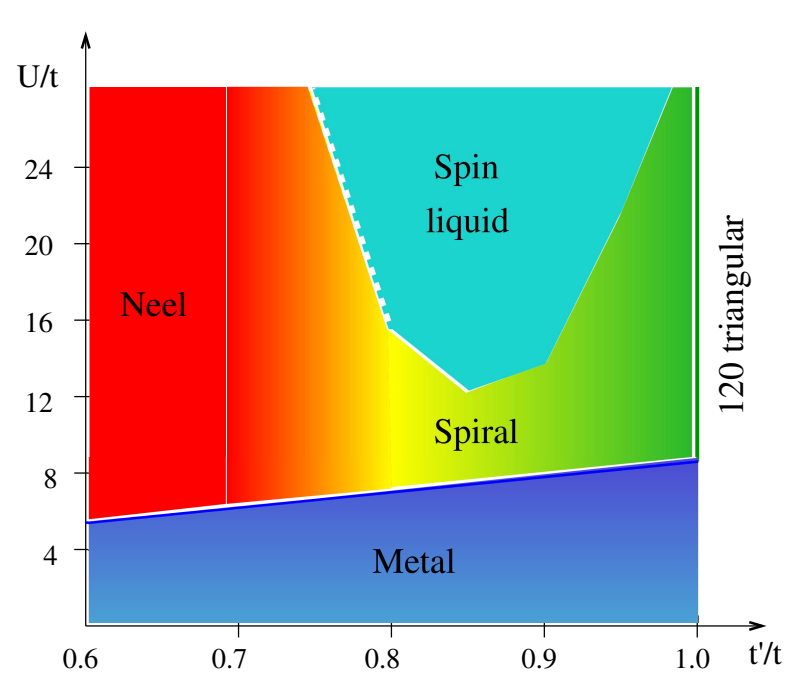

FIG. 6: (Color online) Schematic phase diagram of the Hubbard model on the anisotropic triangular lattice, as obtained by VMC: metal (blue), insulator with magnetic Néel order (red), insulator with spiral magnetic order (gradient redyellow-green), and spin liquid (cyan). The $120^{\circ}$ ordered state with $\theta=2 \pi / 3$ (vertical green line) is stable only for $t^{\prime} / t=1$. The spiral state illustrated with the red-yellow gradient has a pitch angle $\theta$ ranging from $\pi$ to $0.9 \pi$. The border between the spin-liquid and the magnetic state for $t^{\prime} / t<0.8$ (dashed cyan-white line) is only inferred since pitch angles too close to $\pi$ could not be resolved.

$\left.J=4 t^{2} / U\right)$ for the optimal spiral and spin-liquid states as a function of $U / t$ and different values of $t^{\prime} / t$. We find that there is a critical $U / t$ above which the spin-liquid state is energetically favored, while for smaller values of $U / t$ the magnetically ordered state is stabilized. The simple HF energy of the spiral state is also reported for comparison; however, the accuracy of the HF state is not sufficient to study the competition with the spin-liquid state.

Phase diagram - In Fig. 6, we present the final phase diagram. We identify a metallic phase, which is likely not superconductive, 21, 44] and three insulating phases: a phase with commensurate Néel order (i.e., $\theta=\pi$ ), a spiral-order phase with $2 \pi / 3<\theta<\pi$, and a spin-liquid region. Note that the $120^{\circ}$ order with $\theta=2 \pi / 3$ is stable only at the isotropic point $t^{\prime} / t=1$. Within the region $0.7 \lesssim t^{\prime} / t \lesssim 0.8$ the pitch angle is close to $\pi$, and our numerical calculations applied to finite-size clusters cannot resolve the actual value of the optimal $\theta$. Our results indicate that the spin-liquid state is not stable asymptotically close to the isotropic point, for $t^{\prime} \rightarrow t$, at least not for any finite $U / t$. We also point out that the spin liquid described by our variational wave function is gapless at the nodal points $k=( \pm \pi / 2, \pm \pi / 2)$, see Eq. (5). Finally, we would like to mention the fact that, within our present approach, the transition from a spiral state to the spin liquid is always first order. However, we cannot exclude the existence of a continuous transition in the 
exact ground state. In this regard, it would be possible to determine the nature of the transition by considering BCS-spiral uncorrelated states $|\mathrm{BCS}, \mathrm{SP}\rangle$, which are however technically extremely demanding, and beyond the scope of the present study.

Conclusions - By using a state-of-the-art variational approach, we studied the insulating phase of the halffilled Hubbard model on anisotropic triangular lattices with $t^{\prime} / t \leq 1$. Through a combined HF and VMC approach, we showed that spiral states, with non-trivial pitch angles, are stable in the strongly frustrated region. For larger values of interaction a spin-liquid phase emerges. These results open two intriguing possibilities. On one side, the spin-liquid and the spiral phases may be considered two competing phases. In this case, the transition would be expected to be of first order. On the other side, the possibility of a new route towards a non-magnetic correlated state emerges, namely the spin liquid may be considered as an instability emerging from a strongly correlated spiral phase. In this case, a second order transition would be expected. To resolve this question one would need to consider strongly-correlated combined BCS-spiral states, which are however technically very demanding and left for future studies. Finally, in the parameter region of relevance for the reported spin-liquid behavior in organic charge-transfer salts, we also find the spin-liquid phase to be the most stable. Regarding spiral order, to our knowledge no experimental evidence for this phase has been reported for organic charge-transfer salts. It would be desirable to search for such a state, especially in the context of new orderings observed in these materials. [45]

L.F.T. and H.F. acknowledge the support of the German Science Foundation through the grant SFB/TRR49.

[1] $\mathrm{ET}=\mathrm{BEDT}-\mathrm{TTF}$ stands for bis(ethylenedithio)tetrathiafulvalene and $\mathrm{X}$ is a monovalent anion.

[2] K. Kanoda and R. Kato, Annual Rev. of Condens. Matter Phys. 2, 167 (2011).

[3] B.J. Powell and R.H. McKenzie, Rep. Prog. Phys. 74, 056501 (2011).

[4] T. Ohashi, T. Momoi, H. Tsunetsugu, and N. Kawakami, Prog. Theor. Phys. Suppl. 176, 97 (2008).

[5] K. Miyagawa, A. Kawamoto, Y. Nakazawa, and K. Kanoda, Phys. Rev. Lett. 75, 1174 (1995).

[6] H. Elsinger, J. Wosnitza, S. Wanka, J. Hagel, D. Schweitzer, and W. Strunz, Phys. Rev. Lett. 84, 6098 (2000).

[7] S. Lefebvre, P. Wzietek, S. Brown, C. Bourbonnais, D. Jerome, C. Meziere, M. Fourmigue, and P. Batail, Phys. Rev. Lett. 85, 5420 (2000).

[8] P. Limelette, P. Wzietek, S. Florens, A. Georges, T.A. Costi, C. Pasquier, D. Jerome, C. Meziere, and P. Batail, Phys. Rev. Lett. 91, 016401 (2003).

[9] Y. Shimizu, K. Miyagawa, K. Kanoda, M. Maesato, and G. Saito, Phys. Rev. Lett. 91, 107001 (2003).
[10] R.S. Manna, M. de Souza, A. Brühl, J.A. Schlueter, and M. Lang, Phys. Rev. Lett. 104, 016403 (2010).

[11] Me stands for methyl, Et stands for ethyl, Pn denotes a pnictogen atom and dmit is 1,3-dithiole-2-thione-4,5dithiolate.

[12] J.-I. Yamaura, A. Nakao, and R. Kato, J. Phys. Soc. Jpn. 73, 976 (2004).

[13] Y. Shimizu, H. Akimoto, H. Tsujii, A. Tajima, and R. Kato, Phys. Rev. Lett. 99, 256403 (2007).

[14] H.C. Kandpal, I. Opahle, Y.-Z. Zhang, H.O. Jeschke, and R. Valenti, Phys. Rev. Lett. 103, 067004 (2009).

[15] K. Nakamura, Y. Yoshimoto, T. Kosugi, R. Arita, and M. Imada, J. Phys. Soc. Jpn. 78, 083710 (2009).

[16] E.P. Scriven and B.J. Powell, Phys. Rev. Lett. 109, 097206 (2012).

[17] K. Nakamura, Y. Yoshimoto, and M. Imada, arXiv:1208.3954 (2012).

[18] H. Morita, S. Watanabe, and M. Imada, J. Phys. Soc. Jpn. 71, 2109 (2002).

[19] B. Kyung and A.-M. S. Tremblay, Phys. Rev. Lett. 97, 046402 (2006).

[20] R.T. Clay, H. Li, and S. Mazumdar, Phys. Rev. Lett. 101, 166403 (2008).

[21] L.F. Tocchio, A. Parola, C. Gros, and F. Becca, Phys. Rev. B 80, 064419 (2009).

[22] H.-Y. Yang, A.M. Läuchli, F. Mila, and K.P. Schmidt, Phys. Rev. Lett. 105, 267204 (2010).

[23] A.E. Antipov, A.N. Rubtsov, M.I. Katsnelson, and A.I. Lichtenstein, Phys. Rev. B 83, 115126 (2011).

[24] P. Sahebsara and D. Sénéchal, Phys. Rev. Lett. 100, 136402 (2008).

[25] T. Yoshioka, A. Koga, and N. Kawakami, Phys. Rev. Lett. 103, 036401 (2009).

[26] H.R. Krishnamurthy, C. Jayaprakash, S. Sarker, and W. Wenzel, Phys. Rev. Lett. 64, 950 (1990).

[27] C. Pinettes and C. Lacroix, Solid State Commun. 85, 565 (1993).

[28] M. Inui and P.B. Littlewood, Phys. Rev. B 44, 4415 (1991).

[29] B.J. Powell and R.H. McKenzie, Phys. Rev. Lett. 98, 027005 (2007).

[30] Z. Weihong, R.H. McKenzie, and R.R.P. Singh, Phys. Rev. B 59, 14367 (1999).

[31] J. Reuther and R. Thomale, Phys. Rev. B 83, 024402 (2011).

[32] P. Hauke, T. Roscilde, V. Murg, J.I. Cirac, and R. Schmied, New J. Phys. 13, 075017 (2011).

[33] A. Weichselbaum and S.R. White, Phys. Rev. B 84, 245130 (2011).

[34] T. Ohashi, T. Momoi, H. Tsunetsugu, and N. Kawakami, Phys. Rev. Lett. 100, 076402 (2008).

[35] L.F. Tocchio, F. Becca, and C. Gros, Phys. Rev. B 83, 195138 (2011).

[36] F. Becca, M. Capone, and S. Sorella, Phys. Rev. B 62, 12700 (2000).

[37] C. Gros, Phys. Rev. B 38, 931(R) (1988).

[38] F.C. Zhang, C. Gros, T.M. Rice, and H. Shiba, Supercond. Sci. Technol. 1, 36 (1988).

[39] C. Gros, Annals of Physics 189, 53 (1989).

[40] B. Edegger, V.N. Muthukumar, and C. Gros, Adv. Phys. 56, 927 (2007).

[41] M. Capello, F. Becca, M. Fabrizio, S. Sorella, and E. Tosatti, Phys. Rev. Lett. 94, 026406 (2005).

[42] J. Liu, J. Schmalian, and N. Trivedi, Phys. Rev. Lett. 
94, 127003 (2005).

[43] T. Watanabe, H. Yokoyama, Y. Tanaka, and J. Inoue, Phys. Rev. B 77, 214505 (2008).

[44] S. Dayal, R.T. Clay, and S. Mazumdar, Phys. Rev. B 85, 165141 (2012).
[45] P. Lunkenheimer, J. Müller, S. Krohns, F. Schrettle, A. Loidl, B. Hartmann, R. Rommel, M. de Souza, C. Hotta, J.A. Schlueter, and M. Lang, Nature Materials 11, 755 (2012). 